\title{
Numerical Simulation for Hydrodynamic Characteristics of Twin Skeg Container Vessel
}

\author{
A. Priyanto, Y. M. Ahmed, Zulhazazi, and Sunarsih
}

\begin{abstract}
Hydrodynamic characteristics of twin skeg container vessel at different grid refinement and constant speeds were explored using Computational Fluid Dynamics (CFD). RANSE code Ansys CFX fitted with Shear Stress Transport (SST) turbulence model was used to carry out numerical simulations for the hull form of the twin skeg vessel model. Grid generator ICEM CFD was used to build hybrid grids for RANSE code solver. Set with Volume of Fluid (VOF) method, the program was utilised to capture the free surface flow around the hull. With emphasis given to the effect of mesh resolution on resistance, wave cut around hull and wave profile generated, comparison among different cases of grid refinement shows promising results.
\end{abstract}

Index Terms-Twin skeg vessel, CFD, free surface, CFX, resistance, numerical.

\section{INTRODUCTION}

In the study of hull form design, ship flow analysis using CFD appears to have good agreements, at least qualitatively, with the model tests at towing tank. The method is applicable to analyse ship resistance and propulsion as well as assessment of ship manoeuvring performance. Among past researches are assessment and prediction of ship self-propulsion performance [1], [2] and analysis of bare hull, rudder and propeller [3].

Simulation of free surface flow around ship hull form based on NSE is usually carried out by interface-tracking method with a moving mesh [4], [5] and interface-capturing method with VOF [6], [7]. Mesh elements in the first method move over an underlying fixed Eulerian grid to track a free surface flow pattern around ship hull. This approach is applicable for moving boundary problems though special treatments are required for simulating problems of large deformation such as breaking waves. For the latter method, both air and water are considered in the simulation and treated as two effective fluids. Here, numerical grids are fixed in space and predication of free surface location is achieved by solving additional transport equation.

Exact number of grid resolutions which is crucial to control the optimum computational effort required for the simulation is best determined by analysing the characteristic of the cases provided. In a study performed by [8], three grid

Manuscript received May 10, 2015; revised July 16, 2015. This work was supported in part by the Malaysian Ministry of Education under Grant 4L115.

A. Priyanto and Y. M. Ahmed are with the Marine Technology Centre, Faculty of Mechanical Engineering, Universiti Teknologi Malaysia, 81300 UTM Skudai, Johor Bahru, Malaysia (e-mail: agoes@mail.fkm.utm.my, yasser@mail.fkm.utm.my).

Zulhazazi and Sunarsih are with the Faculty of Mechanical Engineering, Universiti Teknologi Malaysia, 81300 UTM Skudai, Johor Bahru, Malaysia (e-mail: zulhazazi@gmail.com, bekhairunnisa@gmail.com). resolution cases were used to examine hydrodynamic characteristics of a single skeg container ship at constant Froude number. Previously, [9] employed three grid resolution cases on twin skeg LNG ship at the same condition.

Considering the ever increasing size of container ship, further exploration of twin skeg fitted with twin screw propeller instead of a single one as to trade off the propulsive efficiency of the propulsion is significantly required. Urged by such necessity, this study investigates the hydrodynamic characteristics of a container ship model fitted with twin skeg. Six cases of grid resolutions were considered to facilitate the selection of the optimum number of grid resolutions.

\section{DESCRIPTION OF MODEL}

The study explored single skeg container ship which is modified to be twin skeg using Rhinoceros 5.0 by referring to the design of [8]. The distance of the skeg is about $45.5 \%$ of ship breadth from longitudinal centreline. Table I shows the particulars of the model employed.

TABLE I: PRINCIPLE DIMENSION OF THE DESIGN

\begin{tabular}{ccccc}
\hline Length & Beam & Draft & Wetted Surface Area & $\begin{array}{c}\text { Volume } \\
\text { displace }\end{array}$ \\
\hline $3.84 \mathrm{~m}$ & $0.644 \mathrm{~m}$ & $0.211 \mathrm{~m}$ & $3.128 \mathrm{~m}^{2}$ & $0.286 \mathrm{~m}^{3}$ \\
\hline
\end{tabular}

\section{Mathematical Model}

Mathematical description of free surface flow in CFX is based on the homogenous multiphase Eulerian-Eulerian fluid approach. Both fluids (water and air) in this approach share the same velocity and other relevant fields such as temperature, turbulence, etc., which is separated by a distinct resolvable interface. The local equations governing the motion of unsteady, viscous and incompressible fluid (either liquid or gas) namely Navier-Stokes is given as

$$
\begin{gathered}
\frac{\partial}{\partial t}(\rho)+\frac{\partial}{\partial x_{i}}\left(\rho u_{i}\right)=0.0 \\
\frac{\partial}{\partial t}\left(\rho u_{i}\right)+\frac{\partial}{\partial x_{i}}\left(\rho u_{i} u_{j}\right)=-\frac{\partial \rho}{\partial x_{i}}+\frac{\partial}{\partial x_{i}}\left(-\rho \overline{u_{i}^{\prime} u_{j}^{\prime}}\right) \\
+\frac{\partial}{\partial x_{i}}\left\lfloor\mu\left(\frac{\partial u_{i}}{\partial x_{j}}+\frac{\partial u_{j}}{\partial x_{i}}-\frac{2}{3} \partial_{i j} \frac{\partial u_{l}}{\partial x_{l}}\right)\right\rfloor
\end{gathered}
$$

where

$$
\left(-\rho \overline{u_{i}^{\prime} u_{j}^{\prime}}\right)=\mu_{t}\left(\frac{\partial u_{i}}{\partial x_{j}}+\frac{\partial u_{j}}{\partial x_{i}}\right)-\frac{2}{3}\left(\rho k+\mu_{t} \frac{\partial u_{i}}{\partial x_{i}}\right) \partial_{i j}
$$


and

$$
\rho=\sum_{\alpha=1}^{2} r_{\alpha} \rho_{\alpha} ; \mu=\sum_{\alpha=1}^{2} r_{\alpha} u_{\alpha} ; \sum_{\alpha=1}^{2} r_{\alpha}=1
$$

The SST turbulent model was used in CFX code formulated by

$$
\begin{gathered}
\frac{\partial(\rho k)}{\partial t}+\frac{\partial}{\partial x_{i}}\left(\rho k u_{i}\right)=\frac{\partial}{\partial x_{j}}\left(\Gamma_{k} \frac{\partial k}{\partial x_{j}}\right)+G_{k}-Y_{k} \\
\frac{\partial(\rho \omega)}{\partial t}+\frac{\partial}{\partial x_{t}}\left(\rho \omega u_{i}\right)=\frac{\partial}{\partial x_{j}}\left(\Gamma \omega \frac{\partial k}{\partial x_{j}}\right)+G_{\omega}-Y_{\omega}+D_{\omega}
\end{gathered}
$$

where $\Gamma k$ and $\Gamma \omega, G k$ and $G \omega$, and $Y k$ and $Y \omega$ respectively represent the effective diffusivity for $k$ and $\omega$, generation of turbulence kinetic energy and $\omega$ due to mean velocity gradients, and dissipation of $k$ and $\omega$ due to turbulence respectively. Meanwhile $D \omega$ represents the cross-diffusion term.

\section{COMPUTATIONAL GRID}

A commercial code, RANSE (ICEM CFD) was used to generate hybrid mesh and the distance of the first grid point off the ship surface is maintained for each case, $y+\approx 8$ that is within a log-law region. The computational domain of the model which is extended to $1.5 \mathrm{~L}$ in front, $2.5 \mathrm{~L}$ behind, $1.5 \mathrm{~L}$ to the side and 1.2L under the keel of the ship model has been meshed with unstructured tetrahedral mesh elements of the same size for the six cases i.e. 385098. The air layer is extended to $0.125 \mathrm{~L}$ above the still water surface [10]. Since the main concern of the work was to investigate the resistance of the twin skeg model with the increase of grid resolution, the surface element size of the model was changed according to the scale factor.

Table II shows number of mesh elements used for each case. Different element size is used for each case and unstructured tetrahedral is built in the region around the model. The smaller the size of element used the higher the model element number produced. Furthermore, small size of element used on and near the ship hull surface is to obtain accurate values of pressure force. In the meantime, prism layer is built around the model with total of five layers to produce better free surface effect. The height of the prism layer is kept constant for each case in order to maintain the $y+$ value.

TABLE II: NumBER UNSTRUCTURED AND TOTAL MESH ELEMENTS USED FOR EVERY CASE

\begin{tabular}{|c|c|c|}
\hline Case & Model & Total \\
\hline 1 & 1678813 & 2063911 \\
\hline 2 & 2560444 & 2945542 \\
\hline 3 & 3818997 & 4204095 \\
\hline 4 & 4758121 & 5143219 \\
\hline 5 & 5659270 & 6044368 \\
\hline 6 & 6883022 & 7268120 \\
\hline
\end{tabular}

\section{COMPUtational Method AND Boundary CONDITION}

The current study considered the flat bottom as sea bed without natural irregularities and excluded the dynamic mesh hence treated the ship model without propeller influence. The computation is run with the effect of free surface and surface without wave and current. The water region width is set to fourth of ship lengths as to eliminate the wall effect and no-slip boundary condition is imposed on the hull surface. The static pressure and initial location of the free surface are accordingly defined by the function of water volume fraction at the outlet boundary and volume fraction of water and air at both inlet and outlet boundaries. The scalable wall function is used with turbulence model while the reference pressure is set to the atmospheric pressure. Only the starboard side of the hull model is considered with CFX due to symmetric properties of the problem under consideration. The flow is considered steady in ANSYS CFX calculations with utilisation of finite volume method and high resolution numerical scheme for discretization process and advection terms respectively. The pressure and velocity are correspondingly interpolated using a linear and trilinier numerical schemes. Root mean square (RMS) criterion with a residual target value of $1 \times 10-05$ is used to check the convergence of the solutions. The result is generated using processor i7, 4 core hyper threading with $3.6 \mathrm{GHz}$ and $16 \mathrm{~GB}$ RAM.

\section{RESUlts}

Numerical result by RANSE code is collected for the six cases sufficiently meet the convergence criteria which is defined by the residual errors for the continuity smaller than $1 \times 10^{-5}$. All cases is run at $0.154 \mathrm{~m} / \mathrm{s}$ model speed $(20$ knots full scale ship) with grid refinement increased almost systematically about $6-7 \%$ as to see the pattern of the resistance produced. The grid convergence of the computed frictional resistance force $R f$, pressure resistance $R p$ and total resistance $R t$ which is computed in the CFD is shown in Table III and its correspond coefficient $C f, C p$ and $C t$ is tabulated in Table IV. The coefficients are derived using the formula of $C p=R p / 0.5 \rho \mathrm{Sv}^{2}$ and $C f=R f / 0.5 \rho \mathrm{Sv}^{2}$ by CFX at the defined ship model speed.

TABLE III: NUMERICAL RESISTANCE COMPONENT

\begin{tabular}{llll}
\hline \multirow{2}{*}{ Case } & \multicolumn{3}{l}{ Resistance component } \\
\cline { 2 - 4 } & $R f$ & $R p$ & $R t$ \\
\hline Case 1 & 7.305 & 10.407 & 17.712 \\
Case 2 & 7.144 & 10.393 & 17.537 \\
Case 3 & 6.995 & 10.357 & 17.352 \\
Case 4 & 6.836 & 10.441 & 17.277 \\
Case 5 & 6.708 & 10.494 & 17.202 \\
Case 6 & 6.720 & 10.532 & 17.252 \\
\hline
\end{tabular}

TABLE IV: NUMERICAL RESISTANCE COEFFICIENT

\begin{tabular}{rccc}
\hline \multirow{2}{*}{ Case } & \multicolumn{3}{c}{ Resistance coefficient } \\
\cline { 2 - 4 } & $C f$ & $C p$ & $C t$ \\
\hline Case 1 & $2.198 \mathrm{E}-03$ & $3.131 \mathrm{E}-03$ & $5.328 \mathrm{E}-03$ \\
Case 2 & $2.149 \mathrm{E}-03$ & $3.127 \mathrm{E}-03$ & $5.276 \mathrm{E}-03$ \\
Case 3 & $2.104 \mathrm{E}-03$ & $3.116 \mathrm{E}-03$ & $5.220 \mathrm{E}-03$ \\
Case 4 & $2.056 \mathrm{E}-03$ & $3.141 \mathrm{E}-03$ & $5.197 \mathrm{E}-03$ \\
Case 5 & $2.018 \mathrm{E}-03$ & $3.157 \mathrm{E}-03$ & $5.175 \mathrm{E}-03$ \\
Case 6 & $2.022 \mathrm{E}-03$ & $3.168 \mathrm{E}-03$ & $5.190 \mathrm{E}-03$ \\
\hline
\end{tabular}

The result as illustrated in Fig. 1 clearly shows that the value of $\mathrm{Ct}$ is decreasing with the increase of grid resolution. As there is no significant change from case 4 to case 6 , it is 
deduced that the grid resolution of case 4 is qualitatively sufficient for practical use of other speeds and conditions as well as for another study. However, comparatively the highest wave cut of the current model presents at bulbous bow region.

Fig. 2 compares the characteristic of wave contour $(\mathrm{z})$ around the ship model at $5.076 \mathrm{~m}$ depth measured from the bottom wall of the computational domain to the draft of ship model. It can be seen that the characteristic differs greatly according to the grid refinement which is likely affected by the surface mesh of the ship hull. The wave contour generated by the current model seems to be higher at bulbous bow and after the stern which is a bit different from the highlight given by [8] for the similar vessel type of single skeg container vessel which produced higher wave contour only at the stern part.

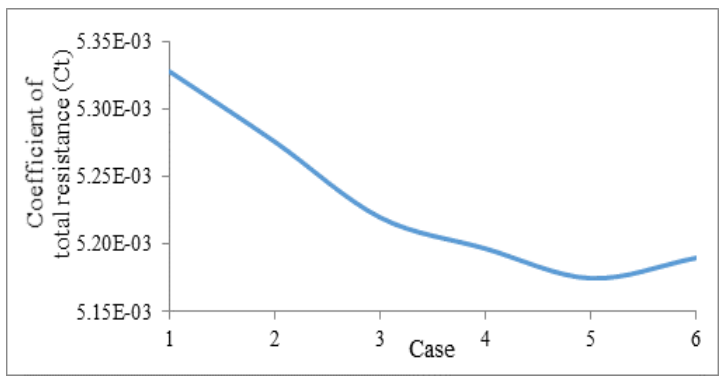

Fig. 1. Coefficient of total resistance for different cases.

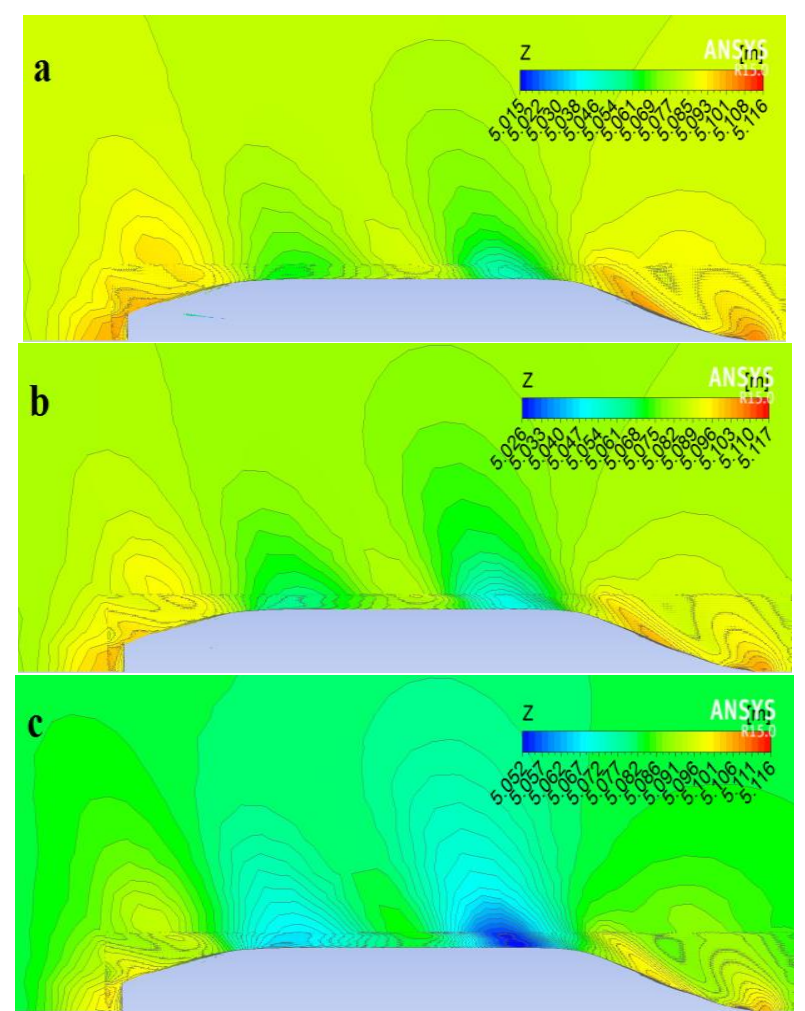

Fig. 2. Comparison of wave contour around (a) case 2, (b) case 4, and (c) case 6.

Fig. 3 compares the wave profiles on the ship hull at a particular ship model speed of $0.0143 \mathrm{~m} / \mathrm{s}$. The profile exhibits that the height of the wave crest is increased accordingly to grid refinement at the fore part and $1.8 \mathrm{~L}$ to $1.5 \mathrm{~L}$ region but not tremendously changed at the bulbous bow. At $1.8 \mathrm{~L}$ to $1.5 \mathrm{~L}$, the flow went through the curvature region before meeting the parallel mid body.
As for case 1 which $\mathrm{m}$ use coarse mesh elements, the wave cut pattern is a lot different from other cases due to less detail of grid size at the surface element mesh though calculated pressure contours similar to other cases are generated in such case due to the readability of the code even in finer meshes as for case 2 and case 4 . Fig. 4 provides evidence of the pressure distribution similarity of the two cases. However, grid refinement of case 4 is preferred by trading off the resistance coefficient yielded.

Comparison of the numerical results shows a good agreement for all cases. As detected by Ansys CFX, high pressure regions are the bow and stern while the low pressure is the hull free surface. Higher pressure at bulbous bow and along the keel to the bottom of skeg is formed by the flow pattern affected by the hull geometry. As the geometry above the keel to free surface region is better thus results in better flow pattern, the pressure decreases accordingly. Remarkably, the code well detected the pressure distribution at draft region based on the effect of the hull wave pattern.

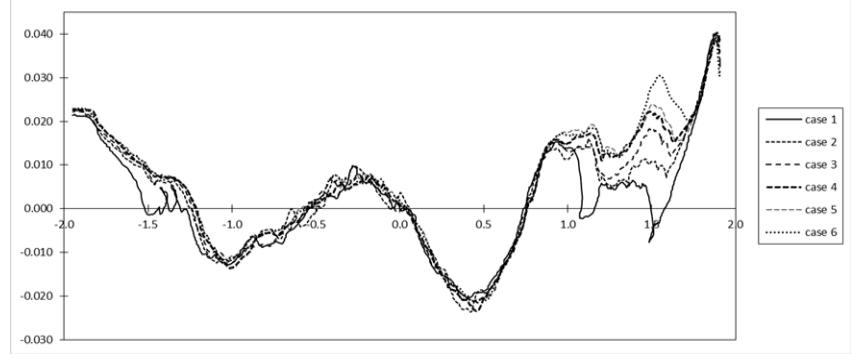

Fig. 3. Wave cut along the hull for all cases at $0.0143 \mathrm{~m} / \mathrm{s}$.

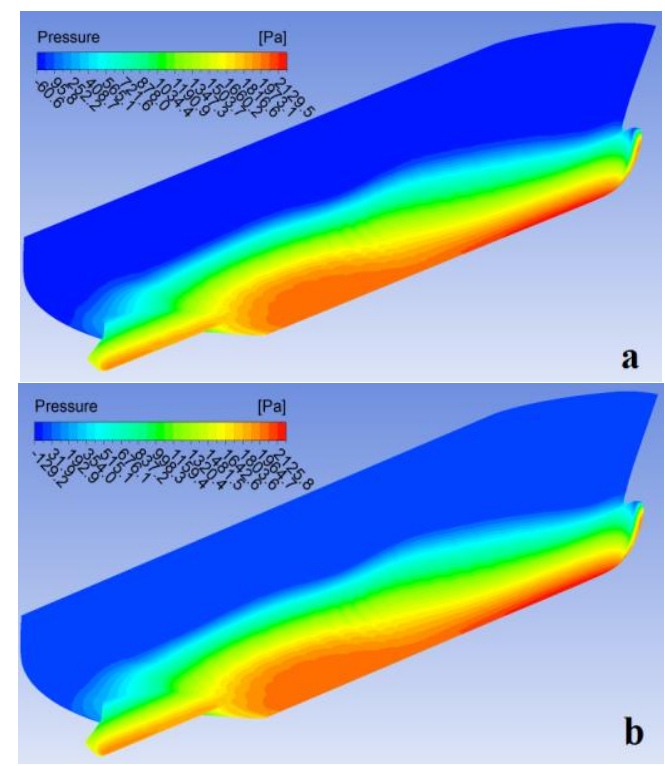

Fig. 4. Pressure contour on twin skeg hull for (a) case 2 and (b) case 4.

\section{CONCLUSION}

The turbulent free surface flow around the hull of the twin skeg has been simulated numerically using the finite volume RANSE code Ansys CFX to get more understanding about the hydrodynamics characteristics of the twin skeg model. Shear stress transient (SST) employed in the numerical analysis reveals that all cases meet the convergence criteria as the residual target is achieved. Most importantly, a suitable grid refinement applicable for other studies such as using of the similar model as well as evaluation of the impact of twin 
skeg fitting to the flow pattern around the stern has been defined thoroughly.

\section{REFERENCES}

[1] J. Kim, I. R. Park, K. S. Kim, and S. H. Van, "RANS computation of turbulent free surface flow around a self-propelled KLNG carrier," $J$ SNAK, vol. 42, no. 6, pp. 583-592, 2005.

[2] H. T. Kim., H. T. Kim, and S. H. Van, "Numerical analysis of flow characteristics of a twin-skeg container ship with variation of stern hull shape," J SNAK, vol. 44, no. 6, pp. 551-563, 2007.

[3] J. J. Kim, K. J. Paik, Y. C. Lee, and H. T. Kim, "A numerical study of turbulent viscous flow around a self-propelled ship," in Proc. the Annual Spring Meeting SNAK., 2006, pp. 548-557.

[4] G. Cowles and L. A. Martinelli, "Viscous multiblock flow solver for free-surface calculation on complex geometries," in Proc. the 22nd Symposium on Naval Hydrodynamics, 1998, pp. 9-14.

[5] T. Li and J. Matusiak, "Simulation of modern surface ships with a wetted transom in a viscous flow," in Proc. the 11th International Offshore and Polar Engineering Conference, 2001, pp. 570-576.

[6] V. Maronnier, M. Picasso, and J. Rappaz, "Numerical simulation free surface flows," Journal of Computational Physics, vol. 155, pp. 439-455, 1999

[7] Y. M. Ahmed, A. Fonfach, and C. G. Soares, "Numerical simulation for the flow around hull of the DTMB 5414 at different speed," International Review of Mechanical Engineering Journal, vol. 4, no. 7, pp. 957-964, 2010.

[8] J. E. Choi, K.-S. Min, J. H. Kim, S. B. Lee, and H. W. Seo, "Resistance and propulsion characteristics of various commercial ships based on CFD results," Ocean Engineering, vol. 37, pp. 549-566, 2010.

[9] D.-W. Park and H.-H. Chun, "Design practice for the stern hull form of a twin skeg ship," Journal of Marine Science Technology, vol. 14, pp. 310-321, 2009.

[10] Y. M. Ahmed. "Numerical simulation for the free surface flow around a complex ship hull form at different Froude numbers," Alexandria Engineering Journal, vol. 50, pp. 229-235, 2011.

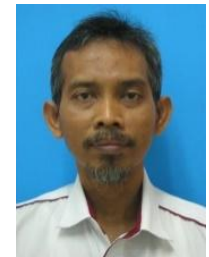

Agoes Priyanto is a senior lecturer at Universiti Teknologi Malaysia. He gives lectures and does researches in the field of self-propulsion, ship resistance and sea keeping and manoeuvring in Marine Technology Centre of Universiti Teknologi Malaysia. He is familiar with validation of propeller performance from CFD using open water test and self-propulsion test. He wishes to develop a new methodology for self-propulsion test using marine diesel engine

simulator.

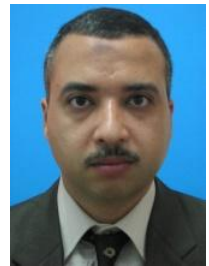

Yasser Mohamed Ahmed is a senior lecturer at Universiti Teknologi Malaysia. He gives lectures and does researches in the field of hydrodynamics, dynamic Marine vehicle, sea keeping and ship resistance propulsion. His specialization is in computational fluid dynamic using various computational method.

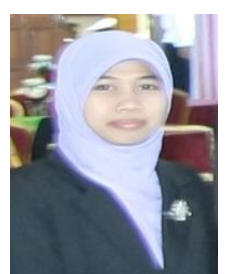

Sunarsih is a post graduate Student at Universiti Teknologi Malaysia. She is currently studying marine propeller hydrodynamics towards development of manoeuvring model in restricted waterways. She has experience in the area of marine propulsion plant, marine engineering and systems, and fuzzy multi criteria decision making method.

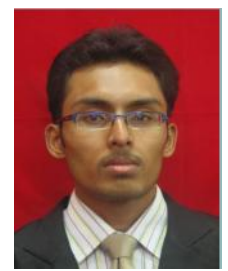

Zulhazazi graduated from Universiti Teknologi Malaysia in mechanical engineering-marine technology. He is a research assistant in the Marine Technology Centre. 